\title{
Observing programs of the Kyiv meridian axial circle equipped with a CCD micrometer
}

\author{
V. Telnyuk-Adamchuk ${ }^{1}$, Yu. Babenko ${ }^{1}$, P. Lazorenko ${ }^{2}$, O. Vertypolokh ${ }^{1}$, O. Molotaj $^{1}$, V. Karbovsky ${ }^{2}$, \\ O. Kovalchuk ${ }^{3}$, V. Andruk ${ }^{2}$, and S. Kasjan ${ }^{1}$
}

1 Astronomical Observatory of the Kyiv National University, Observatornaya 3, 04053 Kyiv-53, Ukraine e-mail: babenko@observ.univ.kiev.ua

2 Main Astronomical Observatory, National Academy of Sciencies of Ukraine, Zabolotnogo 27, 03680 Kyiv-127, Ukraine

3 Mykolaiv Astronomical Observatory, Observatornaya 1, 54030 Mykolaev-MSP, Ukraine

e-mail: al_kov@mao.nikolaev.ua

Received 11 December 2001 / Accepted 8 February 2002

\begin{abstract}
The present status of the Kyiv meridian axial circle after its refurbishment is described. The mounting of a new micrometer with the front-illuminated CCD $1040 \times 1160$ allows to observe stars up to $V=16$ mag. For the stars of 11-14 mag, the internal positional accuracy of a single observation is $0.05-0.10^{\prime \prime}$, and stellar $V$ values are determined with an accuracy of $0.03-0.06$ mag. The project of observations of star fields in the direction of 209 extragalactic radio-sources in declination zone $0+30^{\circ}$ is now in progress. The catalogue of positions, magnitudes and proper motions of secondary reference stars in regions with ICRF sources is expected to be compiled by 2003. Another project under way is a long-term astrometric survey of the equatorial zone to extend the Hipparcos-Tycho frame to fainter stars, and to measure their magnitudes and $V-R$ colours.
\end{abstract}

Key words. instrumentation: miscellaneous - astrometry: reference systems

\section{Introduction}

This paper discusses results of the modernization of the meridian axial circle (MAC) in Kyiv (Scoryk et al. 1989) which was accomplished by means of a new CCD micrometer installation. The use of a CCD instead of the former photoelectric micrometer (Karbovsky et al. 1995) greatly improved astrometric performance of the MAC and allowed us to start new programs of faint star observations. Refurbishment of the MAC followed a similar process which took place at some other meridian circles. It was caused by both an essential progress in the technology of light-sensitive devices used for detection of faint star images, and the release of the Hipparcos and Tycho space catalogues (ESA 1997) having extremely high accuracies of star positions, typically in the limits of 1-30 mas. An improved catalogue, Tycho-2 (Hog et al. 2000) has a high density of stars (60 per square degree on average), which in many cases is sufficient for its use as a reference in small CCD fields. Both factors favoured the

Send offprint requests to: P. Lazorenko, e-mail: gallaz@mao.kiev.ua replacement of photoelectric micrometers by CCD detectors. The use of new micrometers resulted in an increase in the efficiency and accuracy of observations with meridian circles, and considerably fainter magnitudes of stars detected.

The Flagstaff astrometric scanning transit telescope (FASTT) was the first meridian circle equipped with a large CCD and used for routine astrometric observations of stars in scan mode (Stone et al. 1996). Observations with the FASTT have shown that the use of CCD meridian circles is especially efficient for large astrometric surveys. Later, CCD micrometers were mounted on the CAMC (Smart et al. 1999), Valinhos meridian circle in Brazil (Lopes et al. 1999), Mykolaiv AMC (Pinigin et al. 1995), Bordeaux MC (Viateau et al. 1999) and elsewhere. In Kyiv, a new CCD micrometer was mounted on the MAC in February 2000. The micrometer was designed by the Observatory of the National Academy of Sciences, the Kyiv University Observatory, and in collaboration with the Mykolaiv Observatory. After adjustment and alignment, in March 2001 we started observations of secondary reference stars in selected fields with the ICRF sources 
and, later, of the equatorial zone of the sky to make the Hipparcos celestial reference frame (CRF) more dense, and to carry out an astronomical survey.

\section{The instrument}

The MAC (Skoryk et al. 1989) is a meridian refractor $(D=180 \mathrm{~mm}, F=2.3 \mathrm{~m})$ located in the Goloseevo forest, about $10 \mathrm{~km}$ from the centre of Kyiv (Ukraine) at geographic coordinates $\lambda=-2^{\mathrm{h}} 02^{\mathrm{m}} 01^{\mathrm{s}}, \varphi=50^{\circ} 21^{\prime} 55^{\prime \prime}$, at an altitude of $188 \mathrm{~m}$. The telescope was designed as a non-classic instrument of the horizontal type, with all principal elements placed in a horizontal plane. Its tube is oriented in a fixed "east- west" direction, rotates on two high-precision ball-bearings of large diameter, and is aligned with the horizontal axis of the instrument. In front of the objective, a flat glass-ceramic mirror is set rigidly fastened to the MAC tube, forming a $45^{\circ}$ angle with the optical axis. By rotating the tube, the continuation of the optical axis on sky moves along the meridian, thus allowing observations of stars at different zenith angles. A design feature of such a construction, which was very important in the era of wide-angle astrometry, is the ability to control instrumental parameters by means of the long-focus vacuum mark (Lazorenko \& Sergeev 1995). The mark is set on a continuation of the optical axis and is seen simultaneously with the stars through a small opening in the center of the diagonal mirror.

In 1987-1991, fundamental stars and major planets were observed with a visual micrometer. Analysis of these observations and some supplementary studies of the instrument (Bakhonsky 1992; Bakhonsky et al. 1997) have shown sufficient reliability of the MAC design to allow its use for wide-angle astrometry. In 1992, the visual micrometer was replaced by a slit scanning photoelectric micrometer (Karbovsky et al. 1995; Lazorenko et al. 1997) with which positions, $V$ mag and $V-R$ colours of some $927 \mathrm{FK} 4$ stars (Bakhonsky et al. 1998) were measured with a high accuracy.

The new micrometer is based on a front-illuminated CCD Silar ISD017AP with $1040 \times 1160$ pixels. Along its edges the chip contains 54 additional columns of pixels screened from light and used only for calibrations. At a $16 \mathrm{mkm}$ pixel size and a scale of $1.394^{\prime \prime}$ per pixel, the CCD covers a $24.2^{\prime}$ wide strip in declination. The exposure, or the time interval during which equatorial stars cross a matrix, is about $108 \mathrm{~s}$. The electric signal from the CCD after amplification is conversed to a 12-bit digital form. The dark current is $\sim 6 \mathrm{e}^{-} \mathrm{pk}^{-1} \mathrm{~s}^{-1}$, and the readout noise $20 \mathrm{e}^{-}$. A two-stage thermo-battery ensures cooling of the chip to $40^{\circ}$ below the ambient air temperature. Electronics support scan mode of observations and is controlled by the computer Pentium-166 which registers observational information. For long-term storage, information is saved on CD-ROM disks.

To standardize instrumental photometric system, a glass filter with a passband of $480-580 \mathrm{~nm}$ reproducing a Johnson $V$ filter is now used. The brightest images detected with no pixel saturation correspond to stars of $11 \mathrm{mag}(V)$, and the limiting magnitude is about $16(V)$. At $0^{\circ}<\delta<30^{\circ}$ where the CCD scan distortion (Stone et al. 1996) is small, the star images are of symmetric Gaussian shape with $F W H M=2.5$ pk. In future, we intend to use $R$ filter of the Johnson-Morgan extended photometric system which, due to a wider passband of 580-780 nm, will allow us to observe fainter stars and determine their colour indices.

\section{Observing programs}

After the period of adjustments, in March 2001 we started the program of observations of star fields with radiosources being objects of the ICRF. The observational list includes 209 ICRF sources located in declination zone of $0+30^{\circ}$ and taken from the catalogue GAOUA $99 \mathrm{C} 03$ (Molotaj 2000). The purpose of the program is to determine positions, proper motions, magnitudes and $V-R$ colours of faint stars in the direction of radio-sources, thus creating a set of dense local reference frames in the system of the Hipparcos-Tycho optical CRF. Determination of colours is very important since it will allow us to apply corrections for the differential colour refraction. At moderate zenith angles, the amplitude of colour effects of $\sim 20$ mas is quite small, but not can be neglected in precise studies linking optical and radio reference frames (Stone et al. 1999). Colours may be derived two ways: based on $R$ values measured with the MAC, and by using $R$ values of the USNO CCD Astrograph Catalogue (UCAC) having a passband of 579-642 nm (Zacharias et al. 2000).

A catalogue of measured astrometric and photometric data for stars to approximately $16 \mathrm{mag}(V)$ will be built by 2003. Reduction to the ICRF system is made differentially, using the Tycho-2 stars as reference. About 600 strip scans of the sky each of 24.2 (declinations) $\times$ $46.5^{\prime}$ (right ascensions) size $(1040 \times 2000 \mathrm{pk})$ have already been obtained with the $V$ filter, thus most of the fields have a 4 -fold coverage.

The second observing program of the MAC is aimed at densification of the Hipparcos-Tycho reference frame and its extension to fainter magnitudes in the equatorial zone. Also, the program will provide invaluable information on astronomical events registered during observations and which may be interesting for related studies. The program will continue for a lengthy period of time and is intended to be an astrometric survey of the sky for declinations initially restricted by $0+5^{\circ}$, with a 4 -fold center-toedge overlapping of scans. In this program, the telescope operates in the mode of very long scans, unlimited in right ascension, and with easy complete automation of the observational process. Long scans are saved as a consequtive set of short $1040 \times 1024$ strips overlapped by 16 pixels. As noted by Stone et al. (1996), observations in this mode are the most efficient for meridian circles, and allow one to obtain the maximum information for a time unit. The first strip scans of the equatorial zone were obtained in October 2001 with the $V$ filter, while the $R$ filter will 


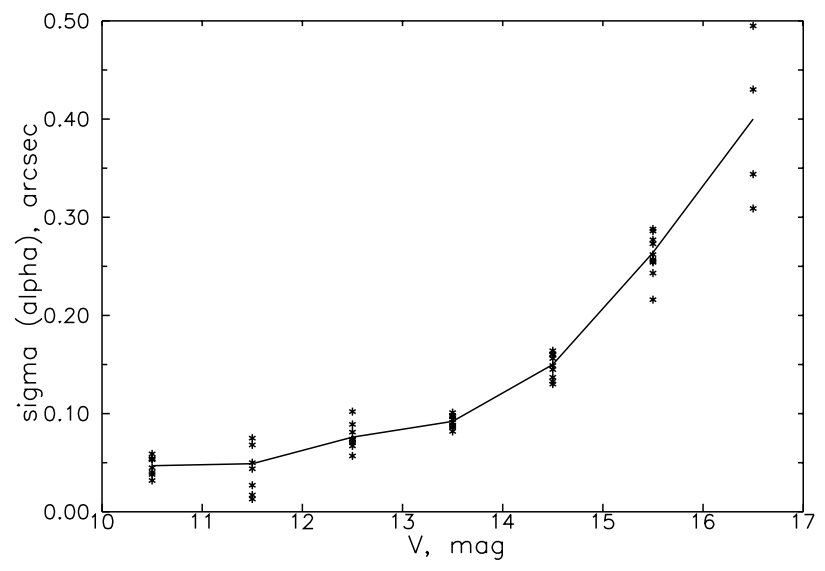

Fig. 1. The internal unit error of star pixel positions $\sigma_{\mathrm{c}}(\alpha)$ in the right ascensions as a function of magnitude $V$. Individual estimates of $\sigma_{\mathrm{c}}(\alpha)$ for each pair of scans (asteriscs) and the mean curve (solid) are shown.

be used later to measure colours of stars. To determine colours, the use of UCAC photometric data is possible. In 16 astrometric calibration regions located along the celestial equator and observed with the FASST, red $R_{\mathrm{F}}$ values are also available (Stone et al. 1999).

\section{Error estimates}

The MIDAS/ROMAFOT software package is used to filter out inhomogeneities typical of the present CCD, locate images, and then derive coordinates and magnitudes of stars. Star images are fitted by a circular Gaussian function.

A tentative analysis of the accuracy of observations with the MAC has been performed with respect to internal errors in two different ways, using: 1) star pixel coordinates derived with a centroiding procedure, and 2) star equatorial positions referred to the ICRF by means of the Tycho- 2 catalogue. For the analysis we used a field with the ICRF J170734.4+014845 which has been scanned for five nights. The number of stars detected in each $1040 \times 2000$ pk field varied from 336 to 460 depending on conditions of observations (atmospheric extinction and sky background).

The first kind of error was analyzed in the following way. After the fitting procedure, the differences of measured star coordinates were formed for each pair of CCD scans; all possible combinations of scan pairs (ten combinations in this case) were considered. These differencies then were corrected for the zero-point shift and the temporal drift of coordinate systems was approximated by a 3- to 4 -order polynomial of time. With these residuals, the estimate of accuracy of the star image measurements $\sigma_{\mathrm{c}}(\alpha)$, $\sigma_{\mathrm{c}}(\delta)$ were computed for each pair of scans and binned in 1 mag intervals.

Photometric data were processed in a similar way, but corrections applied were limited by a constant average difference of intensities in scans caused by changes in the light extinction and background.

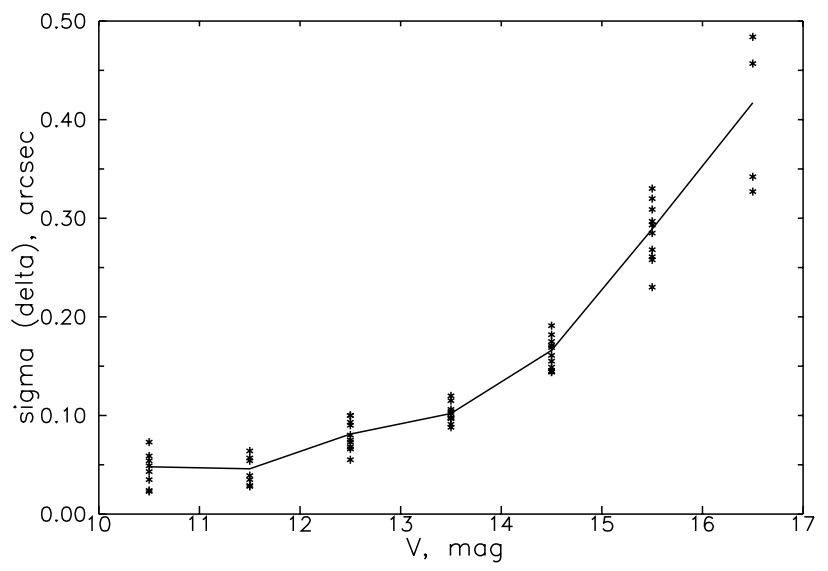

Fig. 2. The same as in Fig. 1 for the errors $\sigma_{\mathrm{c}}(\delta)$ in declinations.

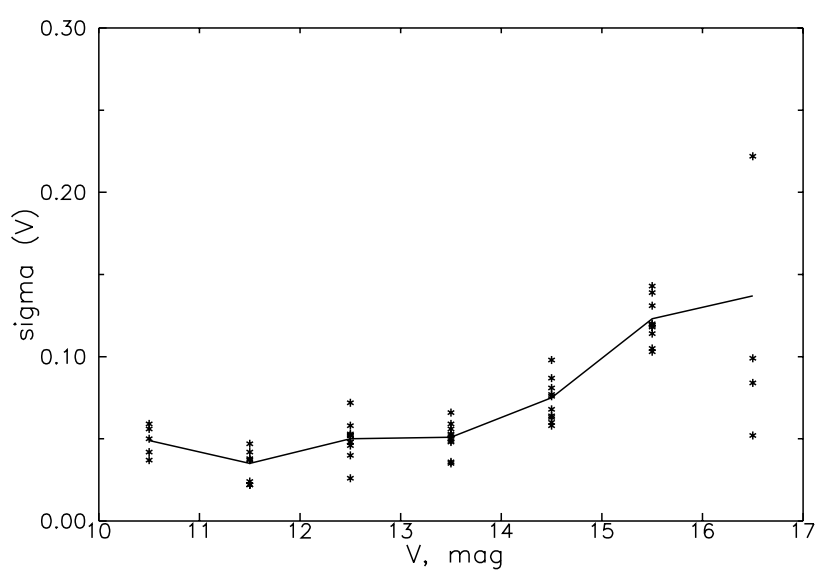

Fig. 3. The same as in Fig. 1 for the errors $\sigma(V)$ in $V$ magnitudes.

In Figs. 1-3, internal errors of one observation $\sigma_{\mathrm{c}}(\alpha)$, $\sigma_{\mathrm{c}}(\delta)$ and $\sigma(V)$ as a function of $V$ mag are shown. The asteriscs correspond to estimates for individual pairs of CCD scans; the solid lines are the weighted averages. Individual pairs presented by less than three stars (bright end of the $V$ axis) were discarded. Typically, the number of stars representing a pair of CCD scans varies from 5-20 (at $V=11-12$ ) to $50-100$ (at $V=14-15)$.

The zero-point of instrumental $V$ values used to plot Figs. 1-3 was found with magnitudes of the Tycho- 2 catalogue (Hog et al. 2000). A limiting magnitude $V=16.0$ for the MAC was estimated from a visual inspection of CCD scans of the open cluster NGC 2264 and other fields containing photometric standards.

The internal positional errors are caused by various sources. First, it is the Poisson photon noise introduced by the star image, the background and CCD readout. Star pixel positions are also affected by changes in scale, refractional anomalies, and instability of the MAC mechanical structure. Internal accuracy is thus a measure of random changes in star pixel positions which occur from frame to frame, and which could not be accounted by the fitting procedure. The estimates of $\sigma_{\mathrm{c}}(\alpha), \sigma_{\mathrm{c}}(\delta)$ therefore correspond to positional errors when the positions are derived 


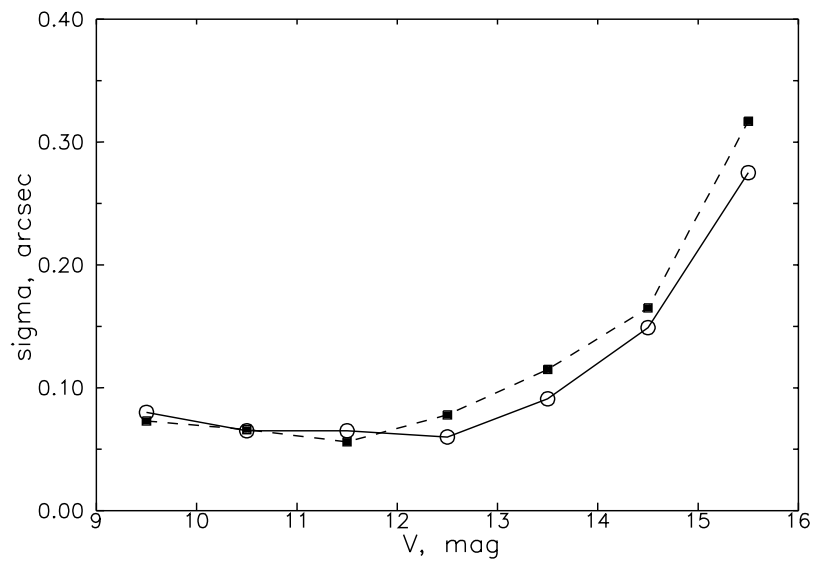

Fig. 4. Internal positional errors in right ascensions $\sigma(\alpha)$ (cirles) and in declinations $\sigma(\delta)$ (squares) as a function of $V$ mag.

with a given reduction model and with a use of a very dense reference catalogue.

Owing to the finite star density, the use of the Tycho2 catalogue as a reference may not allow one to perfectly account for some error components. Also, the Tycho- 2 catalogue is known to have less accurate positions for new objects not contained in the Tycho-1 (Camargo et al. 2001). To estimate a loss in the accuracy introduced by the reduction procedure, we computed equatorial positions of stars in each field. The least square solution was based on a linear transformation with extra temporal polinomials of the 3 -d order. Resulting positional errors $\sigma(\alpha), \sigma(\delta)$ are shown in Fig. 4 and do not differ much from those found above. The star spacing in the Tycho- 2 catalogue is thus sufficient for reduction purposes with a given CCD.

\section{Conclusion}

First observations of stars, and error estimates obtained with a CCD micrometer, allow us to conclude that the MAC may be efficiently used in a number of observing projects, in particular, to link the radio and optical reference frames. A large astrometric survey of the equatorial zone in the two photometric passbands will provide extensive astrometric and photometric data that may serve as a source of information when studying nonstationary events and moving objects. The accuracy of a single position measurement for 11-14 mag stars is 0.05 $0.10^{\prime \prime}$, and the accuracy of photometry is $0.03-0.06 \mathrm{mag}$. At 4-5 fold overlapping of strips, the errors of the catalogue data are expected to be $0.02-0.05^{\prime \prime}$ for positions and 0.02-0.03 for magnitudes.

\section{References}

Bakhonsky, A. 1992, Ph.D. Thesis, Main Astron. Obs., Kyiv Bakhonsky, A., Kostjuchenko, V., \& Lazorenko, P. 1997, Kinemat. Phys. Celest. Bodies, 5, 58

Bakhonsky, A., Karbovsky, V., \& Lazorenko, P. 1998, Kinemat. Phys. Celest. Bodies, 4, 15

Camargo, J. L., Teixeira, R., Benevides-Soares, P., \& Ducourant, C. 2001, A\&A, 375, 308

ESA 1997, The Hipparcos and Tycho catalogues, v.1-17 (Noordwijk: ESA Publ. Division)

Hog, E., Fabricius, C., Makarov, V., et al. 2000, A\&A, 355, L27

Karbovsky, V., Lazorenko, P., \& Sergeev, A. 1995, Kinemat. Phys. Celest. Bodies, 1, 75

Lazorenko, P., \& Sergeev, A. 1995, Astron. Nachr., 316, 131

Lazorenko, P., Bakhonsky, A., Karbovsky, V., \& Sergeev, A. 1997, Kinemat. Phys. Celest. Bodies, 6, 14

Lopes, P. A., Andrei, A. H., Puliaev, S. P., et al. 1999, A\&AS, 136,531

Molotaj, O. 2000, in IVS 2000 General Meeting (Koetzting), ed. N. Vandenberg, \& K. Baver, NASA, Hanover, 338

Pinigin, G., Shulga, A., Fedorov, P., et al. 1995, Astron. and Astrophys. Trans., 8, 161

Scoryk, K., Lazorenko, P., Minyajlo, N., et al. 1989, Kinemat. Phys. Celest. Bodies, 2, 79

Smart, R. L., Bucciarelli, B., Lattauzi, M. G., et al. 1999, A\&A, 348,653

Stone, R. C., Monet, D. G., Monet, A. K., et al. 1996, AJ, 111, 1721

Stone, R. C., Pier, J. R., \& Monet, D. G. 1999, AJ, 118, 2488

Viateau, B., Requieme, Y., Campion, J., et al. 1999, A\&AS, 134,173

Zacharias, N., Urban, S. E., Zacharias, M., et al. 2000, AJ, 120, 2131 